\title{
La influencia del género en la empleabilidad en jóvenes con empleo y sin empleo
}

\author{
IRENe Mengual Alcaraz \\ mengual@uji.es \\ NieVES Fuentes SÁNCHEZ \\ al262229@uji.es \\ M. Alexandra Vass \\ al288986@uji.es \\ Eva Cifre Gallego \\ cifre@uji.es
}

\section{Resumen}

Introducción: De acuerdo con la teoría de roles de género, existirá una segregación horizontal y vertical en función al género que podría estar afectando la empleabilidad del acceso y mantenimiento del empleo de jóvenes menores de 30 años. Por tanto, el objetivo de este estudio es analizar si el género y sus estereotipos asociados afectan de manera diferencial la búsqueda o el mantenimiento de un empleo a este colectivo. Método: Realizamos un estudio de tipo cualitativo formado por dos focus group de jóvenes menores de 30 años con empleo (en activo durante 2 años mínimo) y sin empleo (en situación de desempleo mayor a 6 meses pero con experiencia laboral) respectivamente. Se les pide a los participantes que describan si creen que el género les ha influido en los procesos de búsqueda o mantenimiento de un empleo. Los focus group duraron 2 horas aproximadamente. Posteriormente fueron transcritas y analizadas por 3 jueces diferentes. Resultados: Existencia notoria de empleos estereotipados (menor responsabilidad en empleos femeninos), segregaciones laborales -horizontales y verticales- y diferentes condiciones laborales (las mujeres tenían menor salario, y jornadas parciales, los hombres jornadas completas). Conclusiones: Todavía es visible la segregación estereotipada por género en el proceso de búsqueda y la calidad del empleo. Finalmente, proponemos medidas de acción para obtener la igualdad.

Palabras clave: empleabilidad, género, estereotipos, desempleo.

\section{Abstract}

Introduction: According to the theory of gender roles, there would be a horizontal and vertical segregation related to gender that could be affecting the employability (employment access and maintenance) in young people under 30 years. Therefore, the aim of this study is to analyse if gender and its associated stereotypes are influencing in a different way the search or maintaining of an employment. Method: We performed a qualita- 
tive study based on two focus group of young people less than 30 years, with employment (working at least 2 years) and without it (unemployed higher to 6 months). Participants were asked to describe if they believe that gender has influenced them in this process of searching or maintenance of employment. Focus group lasted about two hours. Later on, they were transcribed and analysed by 3 different judges. Results: Notorious existence of stereotyped jobs (less responsibility in female jobs), work horizontal and vertical segregations and different working conditions (women had lower pay for women part-time jobs; men had full-time jobs). Conclusions: Stereotyped segregation based on gender is still visible in the process of job searching, and its quality. Finally, we propose some steps for actions looking for equality.

Keywords: employability, gender, stereotypes, unemployment.

\section{Introducción}

En el contexto económico actual el empleo y la empleabilidad suponen facetas destacables de la vida del individuo. Como muestran los datos del Instituto Nacional de Estadística (INE, 2015), la inestabilidad laboral supone un desafío en la trayectoria profesional. A pesar de que la tasa de paro en España en el primer trimestre de 2015 desciende respecto al último cuarto del año anterior en 13.100 personas, el número de desempleados/as todavía asciende a casi 5,5 millones, de los cuales los jóvenes menores a 30 años de edad representan el 14,40 $\%$ hombres y $12,51 \%$ mujeres (INE, 23 de abril de 2015).

La satisfacción en el ámbito laboral forma parte de los indicadores de bienestar del individuo en la sociedad actual. Según la Organización Mundial de la Salud (OMS), «la salud es un estado de completo bienestar físico, mental y social, y no solamente la ausencia de afecciones o enfermedades» (OMS, 1948). Un componente que influye en la salud del individuo es, pues, el bienestar emocional. Se tienen que considerar las condiciones laborales para mantener el equilibrio de tipo afectivo y evitar posibles situaciones de exclusión. El estado de limitación más común es el de discriminación tratando a las personas «de forma diferente y menos favorable debido a determinadas características como el sexo, el color de la piel, su religión, ideas políticas u origen social, con independencia de los requerimientos del trabajo» (OIT, 2003, pp. 15-16).

Partiendo desde el fundamento biológico, Fausto Sterling (1994) no encontró diferencias comportamentales entre hombres y mujeres de edad adulta, pero los resultados apuntaron a desigualdades resultantes de la experiencia, ya que indicó que las distintas sociedades influyen sobre las personas. Referido a las diferencias anatómicas y fisiológicas se utiliza el término de sexo, mientras que al término género se le da uso en cuanto a funciones, papeles y responsabilidades establecidas por la sociedad. Cada individuo ha nacido con su sexo, pero se aprende a ser niña, niño, mujer y hombre a través de la socialización diferenciada (Walker y Barton, 1983).

La asimilación interna del aprendizaje de diferenciación de género conduce a la distinción entre rol de género masculino y rol de género femenino, proceso por el cual los individuos de una sociedad aprenden e incorporan valores y comportamientos asociados al prototipo masculino y femenino, respectivamente. Este proceso de socialización, denominado socialización de género (Kohlberg y Ullian, 1974; Slaby y Frey, 1975) comprende una doble vertiente: la colectiva, que supone la adaptación individual en función de las expectativas de la sociedad, 
y la individual, que supone la interiorización de los roles y estereotipos, y educar por igual a los descendientes. La socialización individual hace casi inabordable la realización de planes de acción equitativos. Los roles de género conservan relación directa con el reparto de tareas entre hombres y mujeres. En Género. Manual de capacitación (FISCR y la Media Luna Roja, 2002) se analizan situaciones cotidianas con participación de las mujeres en tareas domésticas y cuidado de personas mayores, y de los hombres en órganos con toma de decisiones y elevado nivel de responsabilidad. Interviene aquí la condición de estereotipo, enmarcada en el contexto laboral.

El concepto de estereotipos favorece la tendencia a representar al género en grupos homogéneos (por ejemplo, los hombres son unos machistas o las mujeres son unas retorcidas) y para diferenciarlos entre sí (por ejemplo, los hombres suelen ser, por regla general, competentes a diferencia de las mujeres, o las mujeres suelen ser sensibles, mientras que los hombres se caracterizan por su falta de sensibilidad) (Barberá, Martínez Benlloch y Bonilla Campos, 2004).

Los datos recogidos durante el primer trimestre del 2015 (INE) de personas en desempleo muestran diferencias entre géneros dependiendo de diversos factores individuales. Hasta la segunda etapa de educación secundaria el porcentaje de hombres y mujeres en desempleo (menores de 30 años) se muestra equitativo, en cambio cuando hacemos mención a estudios de educación superior observamos que el número de mujeres paradas es mayor (178.700) en comparación con los hombres (127.100). Hay diferencias en el tipo de jornada laboral a tiempo completo (hombres 101,1 y mujeres 68,5$)$ y a tiempo parcial $(62,600$ hombres frente a 87.600 mujeres). También se muestran distinciones según el estado civil: solteros/as (hombres 724,6 y mujeres 584,6 ), casados/as (hombres 34,3 y mujeres 90,8 , siendo más notorio el desnivel en el rango de edad 25-29 años: hombres 31.2 y mujeres 70.9) (INE, abril 2015).

Existen prejuicios contra aquellas mujeres que ocupan un cargo de liderazgo. Estos son consecuencia de la percepción de incongruencia entre el rol femenino y el rol de líder como se apunta en la teoría de congruencia de rol de género propuesta por Eagly y Karau (2002). Constantemente en nuestra sociedad el rol que enfatiza el poder, la competición y la autoridad se relacionan con el género masculino. Y las mujeres se han caracterizado por roles hacía las personas y el apoyo social, y no tanto al poder o el mando (Eagly, 1987; Eagly, Wood, y Diekman, 2000; López-Sáez, 1994; López-Zafra, 1999).

En definitiva, las características que se asocian habitualmente al rol de líder se asocian más frecuentemente al rol de género masculino que al femenino. De este modo, las mujeres experimentan discriminación en puestos de trabajo dominados habitualmente por hombres (Eagly, Karau, y Makhijani, 1995; Heilman, 2001; van Engen y cols., 2001), ya que éstos se perciben como especialmente incongruentes con su rol de género. Este hecho explica el bajo porcentaje de mujeres que asumen un cargo de liderazgo en empresas consideradas propias del género masculino, y la consiguiente segregación de hombres y mujeres en los diferentes puestos de trabajo (Cejka y Eagly, 1999; Jacobs, 1999; López-Zafra y Del Olmo, 1999).

Relacionando la teoría de los roles de género con el aspecto de empleabilidad se produce el fenómeno de segregación de tipo horizontal y vertical, ligada frecuentemente a estereotipos. La segregación horizontal hace referencia a la obtención de un puesto de trabajo feminizado y masculinizado (como por ejemplo enfermera, cocinera, dependienta versus ingeniero, mecánico, obrero), mientras que la segregación vertical se entiende como la imposibilidad de la mujer para acceder a puestos directivos, para ascender en la empresa y obtener mejores condiciones laborales (Van Vianen y Fischer, 2002).

Es un tipo de barrera impuesta por la sociedad y por las mismas mujeres. Algunas de las causas podrían ser los amplios problemas socioeconómicos que presenta la evolución de mujeres y hombres, la perdurabilidad de los estereotipos de género (Espín, 2005; Fleming, 
2005) y otras variables debidas a semejanzas familiares que todavía se mantienen. Las mujeres ocupan el $22 \%$ de los cargos directivos en las empresas españolas de tamaño medio y grande (de 100 a 500 empleados). El porcentaje de mujeres en la directiva de las empresas aumentó 7 puntos porcentuales entre 2004 y 2009, pero se mantiene paralizado desde entonces (Grant Thornton, 2014).

Los estereotipos sexuales se refieren a las creencias generalmente aceptadas y poco cuestionadas que podrían contribuir a cómo los hombres y las mujeres debemos expresar nuestra sexualidad. Así pues, parece ser que el hombre o mujer puede estar afectando de manera diferencial a la empleabilidad de unos y otras, entendiendo por empleabilidad como una condición de preparación para el afrontamiento de las demandas inmediatas o futuras del mercado laboral (Casalli y cols., 1997) que dejar ver los recursos que tiene el/la trabajador y la capacidad que tiene para darles uso en su trabajo. Aunque los grandes datos cuantitativos parecen mostrar que siguen habiendo diferencias, no encontramos estudios que analicen como esta experiencia está afectando, si lo hace, en los jóvenes menores de 30 años, que son el futuro activo laboral del país.

El objetivo de este estudio es analizar si el género y sus estereotipos asociados afectan de manera diferencial a la búsqueda o el mantenimiento de un empleo en el colectivo de jóvenes menores de 30 años, desde un punto de vista experiencial.

\section{Método}

\section{Participantes}

La muestra del proyecto se compone de 11 jóvenes menores de 30 años, empleados y desempleados, organizados en dos focus group.

En el primer focus group participaron 6 jóvenes trabajando en activo durante 2 años mínimo (en activo en el momento de llevar a cabo el experimento) de los cuales 3 eran mujeres y 3 hombres, todas y todos menores de 30 años. Las profesiones de las mujeres eran: docente (trabajando durante 3 cursos escolares), enfermera (desde el 2011) y cocinera (6 meses). Las de los hombres; psicólogo del ámbito de los Recursos Humanos (desde hace 3 años), psicólogo investigador estudiante de doctorado (desde este año académico) y un ingeniero (anteriormente trabajó en una refinería pero a día de hoy llevaba sobre 3 meses como administrativo).

El segundo grupo estuvo compuesto por 5 participantes en desempleo durante mínimo 6 meses y en búsqueda activa en la actualidad, de los cuales 3 eran mujeres y 2 hombres, todas y todos menores de 30 años. Las profesiones de las mujeres eran: estudiante de Traducción e Interpretación (1 año y 2 meses en desempleo), estudiante de Publicidad (en desempleo desde hace 7 meses) y estudiante de Psicología (desempleada desde hace 4 años y 2 meses). En el caso de los hombres: estudiante de Psicología (desempleado desde hace 9 meses) y redactor freelance (es autónomo pero lleva buscando un trabajo por cuenta ajena desde hace 8 meses).

\section{Materiales y procedimiento}

Este estudio ha sido realizado dentro de un proyecto más amplio denominado «Calidad de vida en jóvenes empleados y desempleados: el papel de la regulación emocional», subvencionado por la Universitat Jaume I, y llevado a cabo por el equipo de investigación MPAGER. 
Los y las participantes fueron reclutados mediante anuncios publicados por el recinto universitario y mediante personas conocidas contactadas a través de las redes sociales. Su participación era voluntaria y los datos han sido tratados de forma anónima. No obstante ésta fue remunerada una vez finalizada.

Se organizaron 2 focus group (uno de jóvenes con empleo y otro de jóvenes sin empleo). Al comienzo de cada una de las sesiones la dinamizadora se encargó de explicar a los componentes de los grupos en qué consistía el estudio realizado por el grupo MPAGER. Posteriormente se hicieron auto-presentaciones de las personas asistentes. En el caso de las personas desempleadas, estas explicaron su experiencia laboral hasta ese día y desde cuándo se encontraban en situación de desempleo y en búsqueda activa de éste. $Y$ en el caso de las personas empleadas comentaron cuáles eran sus estudios, los trabajos que habían tenido anteriores y en cuál se encontraban en la actualidad. Una vez finalizadas las presentaciones de los/las participantes se pasó a completar un cuestionario dividido en varias partes (diferentes para cada focus group) que era la base de los focus de manera individual. Posteriormente se hizo una puesta en común, acabando recogiéndose las conclusiones sobre cada una de las preguntas planteadas por parte de la dinamizadora. La duración de las sesiones fue en torno a 2 horas cada una.

La primera parte de los focus group consistió en contestar preguntas sociodemográficas entre las cuales se les preguntaba cuántos miembros componen su unidad familiar, su nivel de estudios y el ingreso del hogar, entre otras. En el caso de las personas sin empleo debían indicar cuánto tiempo (en años y meses) se encontraban en situación de desempleo y cuánto era el tiempo (en años y meses) que se encontraban buscando un trabajo de forma activa.

En cambio en el cuestionario de las personas con empleo debían indicar si trabajaban por cuenta ajena o por cuenta propia, el tipo de contrato que tenían y el nivel de ocupación, entre otros.

La última parte estaba formada por preguntas relacionadas con su experiencia en el ámbito profesional (como están viviendo la situación de desempleo o si sienten inseguridad laboral), si el género influye en éstas y si habían sido víctimas de discriminaciones laborales por cuestión de género o conocían a alguien que lo hubiera sido.

Las preguntas de esta parte para el focus group de las personas empleadas fueron:

- ¿Sientes inseguridad laboral? En caso afirmativo, ¿cómo estás viviendo esta experiencia en el empleo?

- ¿Crees que por ser varón/mujer tu experiencia de inseguridad laboral es diferente a la del otro género? Explícalo.

- ¿Has sufrido alguna vez discriminación de género o conoces alguna situación de discriminación de género hacia un compañero de trabajo? Si se da el caso, explícalo.

- ¿En las experiencias laborales que has tenido y tienes, es el salario el mismo para hombres y mujeres? ¿Por qué crees que ocurre esto?

- ¿Cuáles crees que son tus cualidades que hacen que sigas en dicho puesto?

- Las preguntas para esta parte del focus group de las personas desempleadas:

- ¿Cómo estás viviendo la experiencia de desempleo?

- ¿Crees que por ser varón/mujer tu experiencia es diferente a la del otro género? Explícalo.

- ¿Crees que el tipo de trabajo que buscas está influenciado en alguna medida por ser varón o mujer?

- ¿Sientes que tienes más fácil o más difícil la búsqueda de empleo por ser varón/mujer? Si se da el caso, explícalo. 
- ¿Has sufrido alguna vez discriminación de género o conoces alguna situación de discriminación de género hacia un compañero de trabajo cuando estabas trabajando? Si se da el caso explícalo.

\section{Análisis cualitativo de los datos}

Las entrevistas fueron transcritas y para su análisis se creó una tabla con diversas pautas que se evaluaron en los dos focus group.

Las pautas para el análisis del contenido de la sesión del focus group de las personas en situación de desempleo fueron:

- Si el género influye en la búsqueda de empleo.

- Si el género influye en los puestos de trabajo.

Las pautas para el análisis del contenido de la sesión del focus group de las personas en situación de empleo fueron:

- Inseguridad laboral relacionada con el género.

- Segregación de género en el empleo.

- Género y condiciones laborales.

Tres juezas rellenaron cada una de estas tablas siguiendo las pautas de manera independiente. Posteriormente, se hizo una puesta en común de los resultados para llegar a acuerdos. En caso de que alguna de las juezas no estuviera de acuerdo, se discutió hasta llegar a un acuerdo.

\section{Resultados}

Los resultados obtenidos en la investigación con respecto a las personas con empleo muestran que los hombres tienen experiencia laboral en empleos dirigidos a la producción (por ejemplo, ingeniería), mientras que las mujeres tienen experiencia laboral en empleos dedicados al cuidado y atención de los demás. En el caso de las mujeres podemos hablar de empleos externalizados, es decir, tareas que han llevado a cabo desde hace mucho tiempo dentro de casa y que después se han externalizado (enfermera, maestra y cocinera). Estas diferencias de empleo se conocen como empleos estereotipados.

Por otra parte, las mujeres buscan seguir en el puesto actual y si se les propone mejorar no lo tienen claro. En cambio, los hombres presentan una mayor flexibilidad mental y buscan un desarrollo de carrera. A continuación, se puede ver claro este aspecto. Ante la misma pregunta, podemos ver la respuesta de ambos: M (mujer); V (varón).

Dinamizadora: ¿Piensas en alguna mejora o seguir en el puesto que estas exactamente?

M: Ahí estoy bien.

V: Cosas que me gustaría hacer y que pienso que haré en algún momento, pues intentar conseguir o experiencia internacional o experiencia por lo menos en una empresa que sea multinacional. 
Como hemos dicho anteriormente, existe una segregación horizontal y vertical que podría estar afectando a la empleabilidad a través del acceso y mantenimiento del empleo en función del género. Los participantes empleados de la investigación refieren que sí hay una segregación de género en el empleo. Por ejemplo, afirman lo siguiente:

V: Yo, mi experiencia, con igualdad y con empleo, yo sí, yo vivo la discriminación de las mujeres día a día, constantemente en todos los niveles de la sociedad. Hay casos en los que hay mujeres trabajando a jornada completa que cobran lo mismo que sus compañeros a media jornada. La segregación horizontal es brutal, es decir, empresas en las que te encuentras, departamento de administración, $98 \%$ mujeres, departamento de producción 99 \% hombres o $100 \%$ hombres, es decir si tenemos 50 hombres y 50 mujeres, pero todas la mujeres son administrativas y todos los hombre son de producción.

M: Yo dentro de mi profesión, como maestra o maestro, vamos igual, en el colegio donde yo estoy somos tanto mujeres como hombres y tal, pero si sé de, por ejemplo, mi pareja, está trabajando en una empresa donde no contratan a mujeres, está allí y no contratan a ninguna mujer y ya está o amigas mías que son ingenieras o químicas que también hay empresas que no las contratan, no sé por el tema de tener hijos, que se queden embarazadas, no sé.

Las diferencias de género también se hacen visibles en el salario y las jornadas laborales. Existen casos en los que mujeres a jornada completa cobran lo mismo que hombres a media jornada (el salario en mujeres es del 27 al $35 \%$ menor que en hombres).

Por otro lado, con respecto a los y las participantes sin empleo podemos apreciar que los hombres fomentan el autoempleo mientras que la experiencia laboral de las mujeres son trabajos relacionados con la atención a los demás. En cuanto a la influencia del género en la búsqueda de empleo, uno de los participantes desempleados comenta que no ha visto diferencias, solamente en el caso de las tiendas de ropa (segregación horizontal).

V: En tiendas como de ropa sí que mayormente cogen chicas. Un chico que quiera optar al puesto, no sé, no por el tipo de ropa, sino que llegan a coger solamente a chicas. Y para puestos también del tipo de supermercados hay chicos, pero la gente que está normalmente en cajas son chicas. A lo mejor por el hecho de que están cara al público y prefieren a lo mejor mujeres porque piensan que son... más agradables, más amables, más yo qué sé...

En cuanto a la influencia del género en los puestos de trabajos, ambos géneros opinan lo mismo: existe una segregación vertical que imposibilita a las mujeres acceder a los puestos de alto cargo.

V: Creo que hay chicas que tienen más fácil algunos tipos de puestos. Ahora, a cargos más altos y demás sí que es verdad que a puestos directivos sí que hay minoría en las mujeres.

M: Eh... es que claro, si nos estamos refiriendo a niveles más profesionales y más a perfiles de empresas grandes y empresas con éxito... yo he visto que la mayoría son hombres o varones los que ocupan puestos importantes de trabajo o los que están mejor remunerados. 


\section{Discusión y conclusiones}

Como hemos podido comprobar, el género sí es un factor influyente para el mantenimiento o la búsqueda de empleo. De acuerdo a los datos y los conceptos aportados en la introducción, es posible apreciar una segregación de género, donde mayormente es el género femenino el perjudicado. Esto se contrasta con los resultados obtenidos, ya que hemos encontrado tanto segregación de tipo vertical (problemas a la hora de acceder a un empleo por el género como por ejemplo en el puesto del ámbito de la enfermería), como de tipo horizontal (problemas para ascender a un empleo por ser mujer).

Por otra parte, hemos podido encontrar todavía la existencia de empleos estereotipados, ya sea por los que tienen o han tenido cada participante o por sus experiencias propias contadas en las entrevistas (departamento de administración $98 \%$ mujeres, y departamento de producción $99 \%$ o $100 \%$ hombres).

Sigue siendo también notoria que las características de los empleos entre hombres y mujeres no son equitativas. Por ejemplo, el salario de las mujeres sigue siendo inferior al de los hombres. Esto se puede explicar por la teoría de congruencia de rol de género propuesta por Eagly y Karau (2002). Las mujeres experimentan discriminación en puestos de trabajo dominados habitualmente por hombres (éstas se perciben como incongruentes en su rol de género) y la consiguiente segregación de hombres y mujeres en los diferentes puestos de trabajo.

A pesar de estos resultados obtenidos, debemos recalcar que dichas diferencias van siendo menores conforme avanzan los años; sin embargo, todavía queda mucho trabajo por hacer en un futuro para que éstas sean completamente equitativas. Para que estas medidas sean efectivas y se lleve un control se creó la Red Europea de Mainstreaming de Género, en la que participa el Instituto de la Mujer y para la Igualdad de Oportunidades. Entre las acciones que lleva a cabo esta institución podemos encontrar: informaciones y datos desagregados por sexo, utilización de un lenguaje no sexista, y un enfoque transversal de género, entre otras.

\section{Referencias bibliográficas}

Barberá, E., Matrtínez Benlloch, I., y Bonilla Campos, A. (2004). Psicología y género. Madrid: Prentice Hall.

Casali, A., Rios, L., Teixeira, J. E., y Cortella, M. S. (1997). Empregabilidade e Educação. Novos caminhos no mundo do trabalho. São Paulo: Educ/ Rhodia.

Cejka, M. A., y Eagly, A. H. (1999). Gender stereotypic images of occupations correspond to the sex segregation of employment. Personality and Social Psychology Bulletin, 25, 413423.

Eagly, A. H. (1987). Sex differences in social behavior: A social-role interpretation. Hillsdale, NJ: Erlbaum.

Eagly, A. H., y Karau, S. J. (2002). Role congruity theory of prejudice toward female leaders. Psychological Review, 109, 573-598.

Eagly, A. H., Karau, S. J., y Makhijani, M. G. (1995). Gender and the effectiveness of leaders: A meta-analysis. Psychological Bulletin, 117, 125-145.

Eagly, A. H., Wood, W., y Diekman, A. B. (2000). Social role theory of sex differences and similarities: A current appraisal. En T. Eckes y H. M. Trautmer (eds.), The developmental social psychology of gender (pp. 123-174). Mahwah, NJ: Erlbaum.

Espín (coord.) (2005). Publicitat i sexisme: la mirada crítica de l'alumnat universitari.

Fausto-Sterling, A. (1994). Myths of gender. Biological theories about women and men, $2^{\mathrm{a}}$ ed. Nueva York: BasicBook. 
Fleming, M. (2005). Género y autonomía en la adolescencia: las diferencias entre chicos y chicas aumentan a los dieciséis años. Revista de Investigación Psicoeducativa, 3, 23-35.

Grant Thorntone International Ltd (2014). Grant Thorntone Women in Business Report. España: Grant Thorntone.

García-Retamero, R y López-Zafra E. (2006). Congruencia de rol de género y liderazgo: El papel de las atribuciones causales sobre el éxito y el fracaso. Revista latinoamericana de Psicología, 38, 245-257.

Heilman, M. E. (2001). Description and prescription: How gender stereotypes prevent women's ascent up the organizational ladder. Journal of Social Issues, 57, 657-674.

Instituto Nacional de Estadística [INE] (2015). Extraído desde http://www.ine.es/ el 29 de marzo de 2015.

Jacobs, J. A. (1999). The sex segregation of occupations: Prospects for the 21st century. En G. N. Powell (eds.), Handbook of gender and work (pp. 125-144). Londres: SAGE.

Kohlberg, L., Ullian, D. Z. (1974). Stages in the development of psychosexual concepts and attitudes. En R. C. Friedman, R. M. Richart, y R. L. Wiele (eds.), Sex differences in behavior. Nueva York: Wiley.

López-Sáez, M. (1994). Procesos culturales e individuales implicados en la estereotipia de género. Una aproximación empírica a la elección de carreta. Revista de Psicología Social, 9, 213-230.

López-Zafra, E., y del Olmo, S. M. (1999). Estereotipia de género y liderazgo transformacional en contextos de trabajo típicamente femeninos. Revista de Psicología Social Aplicada, 9, 53-71.

Organización Internacional del Trabajo (2003). Convenio sobre la discriminación en materia de empleo y ocupación, pp. 15-16.

Organización Mundial de la Salud (1948). Preámbulo de la Constitución de la Organización Mundial de la Salud tal y como fue adoptada por la Conferencia Internacional de Salud, Nueva York, 19-22 junio, 1946; firmada el 22 de julio de 1946 por los representantes de 61 estados (archivos oficiales de la OMS, núm. 2, pág. 100) y entrada en vigor el 7 de abril de 1948. Obtenida de http://www.who.int/about/definition/en/print.html.

Slaby, R. G., y Frey, K. S. (1975). Development of gender constancy and attention to same-sex models. Child Development, 47, 849-856.

Van Engen, M. L., van der Leeden, R., y Willemsen, T. M. (2001). Gender, context, and leadership style: A field study. Journal of Occupational and Organizational Psychology, 74, 581 598.

Van Vianen, y Fischer, A. H. (2002). Illuminating the glass ceiling: The role of organizational culture preferences. Journal of Occupational and Organizational Psychology, 75, 315337.

Walker, S., y Barton, L. (eds.) (1983). Gender, class and education. Nueva York: Falmer Press. 